\title{
Comets as Tracers of Solar System Formation and Evolution
}

\author{
Kathleen E. Mandt ${ }^{1}$. Olivier Mousis ${ }^{2}$. \\ Dominique Bockelée-Morvan $^{3}$ - Christopher T. Russell ${ }^{4}$
}

Published online: 2 November 2015

(C) Springer Science+Business Media Dordrecht 2015

The dawn of the space age has brought on a new era for exploring comets. Starting in 1985, a total of ten spacecraft have flown by seven different comets at high relative velocities reaching distances as close as $180 \mathrm{~km}$ from the surface. These missions made a variety of observations, including the first ever in situ measurements of cometary plasma allowing detailed studies of the interaction of the cometary coma and the solar wind. The International Cometary Explorer (ICE) achieved the first cometary flyby passing within $7800 \mathrm{~km}$ of comet 21P/Giacobini-Zinner on 11 September 1985. This same spacecraft passed through the tail of comet 1P/Halley in May 1986, millions of kilometers from the nucleus. Five other spacecraft, Vega $1 \& 2$, Sakigake, Suisei and Giotto also visited comet 1P/Halley in 1986, with Giotto passing through the coma within 600 kilometers of the nucleus on 14 March 1986. Giotto was the first European deep space mission, provided the closest images of comet $1 \mathrm{P} /$ Halley and carried mass spectrometers that measured the composition of ions and neutrals in Halley's coma. Giotto went on to conduct a second flyby of a comet, passing within 200 kilometers from the nucleus of comet 26P/Grigg-Skjellerup on 10 July 1992. The Deep Space 1 mission, primarily designed to test new technology, flew within 2000 kilometers of comet 19P/Borrelly on 22 September 2001. The Stardust mission is the only mission so far to return samples of cometary dust collected during a flyby within 240 kilometers of the surface of comet 81P/Wild 2 on 2 January 2004 and return the samples to Earth for laboratory analysis. Stardust later flew within 180 kilometers of Comet 9P/Tempel 1 on 14 February 2011. The Deep Impact mission sent an impactor into Comet 9P/Tempel 1 on 4 July 2005,

C.T. Russell

ctrussel@igpp.ucla.edu

1 Space Science and Engineering Division, Southwest Research Institute, 6220 Culebra Rd, San Antonio, TX 78238, USA

2 Aix Marseille Université, CNRS, LAM (Laboratoire d'Astrophysique de Marseille) UMR 7326, 13388, Marseille, France

3 LESIA, Observatoire de Paris, LESIA/CNRS, UPMC, Université Paris-Diderot, 92195 Meudon, France

4 Institute of Geophysics and Planetary Physics/UCLA, Los Angeles, CA 90095-1567, USA 
excavating dust and allowing studies of dust and subsurface composition as well as physical properties of the nucleus. This spacecraft was later renamed the EPOXI mission and conducted a flyby of comet 103P/Hartley 2 on 4 November 2010.

Although much was learned from the in situ measurements made during these cometary flybys, they were limited to single high-speed passes through the coma at one point during the comet's orbit around the Sun. In order to provide a more detailed study of a comet, the Rosetta mission was designed to escort a comet during a large portion of its orbit around the Sun and deposit a lander. The Rosetta orbiter has twelve science instruments and the Philae lander has nine additional instruments. Rosetta was named after the famous "Rosetta Stone", an ancient tablet that allowed archeologists to translate Egyptian hieroglyphics providing a breakthrough in understanding of ancient Egyptian civilization. Rosetta was launched in 2004 and arrived at its target, comet 67P/Churyumov-Gerasimenko on 6 August 2014. The spacecraft was the first to ever orbit a comet, obtaining orbits down to $6 \mathrm{~km}$ from the surface. This is also the first mission to successfully land a probe on a comet, which was achieved on 12 November 2014.

In order to prepare the community for the task of placing Rosetta observations within the greater context of using comets as tracers of solar system formation and evolution, a workshop was held in Toulouse, France 1-3 April 2014. More than 100 scientists from 18 different countries participated in this three-day science program, which was comprised of themed sessions, and featured a mixture of invited reviews, invited and contributed talks and posters. Invited speakers included many of the community leaders in cometary science, measurements and technology development. The workshop covered topics ranging from the dynamical and chemical evolution of the solar nebula during formation, to the techniques for measuring the composition of comets. Of particular focus was the role that measurements made by Rosetta could be expected to play in understanding the origin of solar system bodies. The purpose of this special issue is to build upon the results of this workshop, providing a formal record of the state of knowledge leading into the Rosetta mission.

This special volume contains eight papers that cover a range of subjects relating the current understanding of comets to solar system formation and evolution. The first four papers summarize what is known about cometary composition and physical properties of cometary nuclei. Anita Cochran and co-authors in "The composition of comets" review the current state of knowledge of the composition of the gas and dust in the comae of comets based on spectral observations made in numerous wavelength ranges and in situ measurements with mass spectrometers. Observations of how coma gas composition changes with distance from the Sun are also reviewed. Various taxonomic studies are discussed, along with the limitations of taxonomic descriptions. The paper concludes with a description of the Rosetta instrument capabilities for measuring the composition of the gas and dust of the comet 67P/Churyumov-Gerasimenko, as well as the implications for measurements made early in the mission. In "Cometary isotopes measurements", Dominique Bockelée-Morvan and co-authors focus on isotopic measurements made in cometary comae, micrometeorites, interplanetary dust particles and Stardust grains. These measurements cover $\mathrm{D} / \mathrm{H},{ }^{14} \mathrm{~N} /{ }^{15} \mathrm{~N}$, ${ }^{16} \mathrm{O} /{ }^{18} \mathrm{O},{ }^{12} \mathrm{C} /{ }^{13} \mathrm{C}$, and ${ }^{32} \mathrm{~S} /{ }^{34} \mathrm{~S}$ ratios in cometary grains and gases and are critical for evaluating the origin of cometary material, and the physical and chemical conditions in the early Solar Nebula. This paper also presents the capabilities of Rosetta instruments in measuring isotopic ratios. In "The subsurface structure and density of cometary nuclei", Philippe Lamy and co-authors review the knowledge gained of cometary interior structure from groundbased radar studies of the first few meters of cometary nuclei that pass close to Earth. What little information is available suggests significant variation in subsurface properties of cometary nuclei from one comet to the next. Murthy Gudipati and co-authors outline 
how laboratory studies have contributed to the current understanding of comets in "Laboratory studies towards understanding comets". Their paper presents recent advancements in understanding of comets thanks to laboratory studies, outlines the need for new laboratory simulations, and makes predictions for future explorations. Laboratory studies are a critical aspect of cometary science because they help to improve understanding of the cometary interior, surface, coma and tail.

These contributions are followed by two papers that summarize understanding of the chemistry and dynamics involved in the formation of the solar system. In "The composition of the protosolar disk and the formation conditions of comets", Karen Willacy and co-authors summarize our understanding of the composition, structure and evolution of protoplanetary disks by reviewing the chemical processes that began in the parent molecular cloud core and continued through the collapse of that core to form the protosun and the solar nebula, as well as during the evolution of the solar nebula itself. Studies of protoplanetary disks around young stars similar to the proto-Sun are help to understand the evolution of disks similar to the solar nebula while they are in the process of evolving to form their own planetary systems. Through their work we learn about the physical and chemical conditions under which comets formed, and about the types of dynamical processing that shaped the solar system we see today. In "Dynamics of the formation of the solar system", Luke Dones and co-authors discuss the evolution of our understanding of the origins of the three known cometary reservoirs: the Oort Cloud for long-period, the Kuiper Belt for ecliptic comets, and the asteroid belt in the case of main-belt comets.

The last two contributions discuss the implications of cometary studies for understanding the formation and evolution of the solar system. In "On the evolution of comets", Aurélie Guilbert-Lepoutre and co-authors evaluate how the observed physical, chemical and orbital characteristics of comets could have evolved over the more than four billion years due to the time-varying radiative and collisional environment of the solar system. To start, the composition and internal structure of comets inherited from their formation stage may be very diverse. Further evolution of comet nuclei involving some possible processing from radiogenic heating, space weathering and large- and small-scale collisions might have modified their primordial structures and compositions with various degrees. In "Constraints from comets on the formation and volatile acquisition of the planets and satellites", Kathleen Mandt and co-authors evaluate the dual role comets play in understanding the formation and evolution of the solar system. First, the composition of comets provides information about the origin of the giant planets and their moons because they are believed serve as a record of conditions during the early stages of solar system formation. After the solar system formed, the orbits were perturbed allowing them to travel into the inner solar system, impact the planets, and thus contribute to the volatile inventory of planetary atmospheres.

The success of this volume is due to many people; but first of all, the editors wish to thank the authors who had the difficult job of distilling the myriad papers on our current understanding of comets into highly readable articles. The editors also benefited from an excellent group of referees who acted as a test readership, refining the manuscripts provided by the authors. These referees included Jérôme Aléon, the late Claudia J. Alexander to whom this volume is dedicated, Claude Arpigny, Michael Belton, Guillermo Manuel Munoz Caro, Dale P. Cruikshank, Jacques Crovisier, Walter F. Huebner, Wing-Huen Ip, Michiel Hogerheijde, Paul Kamoun, Klaus Pontoppidan, Dina Prialnik, Francois Robert Philippe Rousselot, Diane Wooden.

Equally important has been the strong support this project has received at Springer and the extra effort expended by Jennifer Satten, Jurgita Vanagaite and Nirmala Kumar. At UCLA we were skillfully assisted by Marjorie Sowmendran who acted as the interface between the authors, editors, referees and the publisher. 